\title{
A Fourier Method for the Numerical Solution of Poisson's Equation*
}

\author{
By Gunilla Sköllermo
}

\begin{abstract}
A method for the solution of Poisson's equation in a rectangle, based on the relation between the Fourier coefficients for the solution and those for the right-hand side, is developed. The Fast Fourier Transform is used for the computation and its influence on the accuracy is studied. Error estimates are given and the method is shown to be second order accurate under certain general conditions on the smoothness of the solution. The accuracy is found to be limited by the lack of smoothness of the periodic extension of the inhomogeneous term. Higher order methods are then derived with the aid of special solutions. This reduces the problem to a case with sufficiently smooth data. A comparison of accuracy and efficiency is made between our Fourier method and the Buneman algorithm for the solution of the standard finite difference formulae.
\end{abstract}

1. Introduction. In this paper we will develop a method based on the Fast Fourier Transform, FFT, for the numerical solution of Poisson's equation in a rectangle. The method will be analyzed in detail for the Dirichlet problem. We will also discuss the modifications necessary for the treatment of the Neumann case.

In particular we will discuss Poisson's equation, $\Delta v=G$, in the unit square. In addition, we have boundary conditions which we write as $B v=g$. We will first construct a function $w$ which satisfies the boundary condition, $B w=g$. Thus,

$$
\Delta u=F, \quad B u=0,
$$

where $u=v-w$ and $F=G-\Delta w$. This problem is then solved by approximating $F$ by a linear combination of eigenfunctions to the Laplace operator. Thus, for the $\mathrm{Di}$ richlet problem, we use $\sin (k \pi x) \sin (l \pi y), k, l=1,2, \ldots, N-1$, while we use $\cos (k \pi x) \cos (l \pi y), k, l=0,1,2, \ldots, N-1$ for the Neumann problem. The approximation of $F$ is achieved by the FFT, which amounts to replacing the 2-periodic extension of $F$ by its trigonometric interpolation polynomial on the mesh

$$
\{(x, y) \mid x=i / N, y=j / N, i, j=0, \pm 1, \pm 2, \ldots\} .
$$

An approximation to $u$ is then constructed by dividing each Fourier coefficient to $F$ by the appropriate eigenvalue of the Laplace operator.

In Section 3 we will prove, under certain assumptions on the smoothness of $F$ in the unit square, that the rate of convergence is $O\left(N^{-2}\right)$. Modified versions of the method will give an accuracy of order $N^{-2 p}, p=2,3, \ldots$, for a sufficiently smooth $F$. The modifications are motivated by the fact that the periodic extension of $F$, in general, fails to be sufficiently smooth. The procedure amounts to the introduction of

Received October 28, 1974.

AMS(MOS) subject classifications (1970). Primary 35J05.

Key words and phrases. Poisson's equation, Fast Fourier Transform.

* This work was sponsored in part by the Swedish Institute for Applied Mathematics. 
special solutions to the Poisson equation. With the aid of these, the right-hand side $F$ can be replaced by a function which has a sufficiently smooth periodic extension.

The application of FFT to Poisson's equation has been used by Hockney and others in connection with finite difference techniques, see Hockney [4]. The system of equations which arises from finite difference approximations can in certain cases be solved by a direct method using FFT. This technique can be combined with one or several steps of a block cyclic reduction procedure. An alternative to Hockney's method is the Buneman algorithm (see Buneman [1]), in which the block odd-even reduction is continued until only one block remains. This requires that we have $2^{q}-1$ meshpoints in one direction. This method has been described and analyzed by Buzbee, Golub and Nielson [2] and can be used whenever the problem allows for separation of variables. We have made a few numerical experiments in order to compare the efficiency and accuracy of our Fourier method with Buneman's algorithm. The results indicate that the two methods are equally good. One advantage of the Fourier method is that the number of points need not be restricted if an efficient mixed radix FFT is available.

Since the first version of this report appeared, a report by Rosser [5] has come to our knowledge, in which Poisson's equation is treated by a method very similar to ours. The difference is mainly that Rosser divides the problem into one of finding the solution of Poisson's equation without considering the boundary conditions, and another of solving the Laplace equation with such boundary conditions that the sum of the two solutions is the solution to the original problem. Rosser's procedure can also be modified to handle discontinuous boundary data.

Acknowledgments. I would like to thank Professor H.-O. Kreiss for proposing this problem to me and Professor O. B. Widlund and Dr. O. H. Hald for a detailed criticism of an earlier version of this paper. Thanks are also due to Mrs. Ellen Morrison of Stanford, who made available an efficient Buneman-program for the nine-point formula, and to Dr. B. Fornberg for discussions on the FFT and for a fast program for the real transform.

2. The One-Dimensional Case. We will begin by describing the algorithm in its simplest form, when applied to the problem

$$
\begin{aligned}
u^{\prime \prime}(x) & =F(x), \quad 0<x<1, \\
u(0) & =u(1)=0 .
\end{aligned}
$$

We extend $u(x)$ and $F(x)$ to be odd periodic functions of period 2. Assuming that the extension of $u(x)$ is sufficiently smooth, we can expand it in a uniformly convergent Fourier series,

$$
u(x)=\sum_{k=1}^{\infty} u_{k} \sin (k \pi x),
$$

where $u_{k}=2 \int_{0}^{1} u(x) \sin (k \pi x) d x$. This formula also defines the periodic extension of $u(x)$. Throughout this paper, $u_{k}$ (or $u_{k l}$ ) will denote the exact Fourier coefficient, while $\tilde{u}_{k}$ (or $\tilde{u}_{k l}$ ) denotes the exact coefficient in the interpolating polynomial; and 
$\hat{u}_{k}$ (or $\hat{u}_{k l}$ ) denotes an approximation to $\tilde{u}_{k}$ (or $\tilde{u}_{k l}$ ). Integrating by parts and using Eq. (2.1), we find that

$$
u_{k}=-\frac{2}{\pi^{2} k^{2}} \int_{0}^{1} F(x) \sin (k \pi x) d x \equiv-\frac{F_{k}}{\pi^{2} k^{2}} .
$$

Sampling the values of $F(x)$ at $x_{\nu}=\nu / N, \nu=1, \ldots, N-1$, we apply the FFT-algorithm which corresponds to the usual sine-series to compute approximations $\widetilde{F}_{k}$ to $F_{k}, k=1, \ldots, N-1$; see Cooley, Lewis and Welch [3]. The right-hand side is thus represented in the meshpoints by the trigonometric interpolation polynomial of the 2 periodic extension of $F(x)$,

$$
F(\nu / N)=\sum_{k=1}^{N-1} \widetilde{F}_{k} \sin (k \pi \nu / N)
$$

Correspondingly, the differential equation has the approximate solution

$$
\hat{u}(\nu / N)=\sum_{k=1}^{N-1}-\frac{\widetilde{F}_{k}}{\pi^{2} k^{2}} \sin (k \pi \nu / N)
$$

This function is easily evaluated at $x_{\nu}=\nu / N, \nu=1, \ldots, N-1$, via an inverse FFT.

We can now state the following result:

THEOREM 1. Let $F^{\prime}(x)$ exist and be of bounded variation over the interval $0 \leqslant$ $x \leqslant 1$. Then the Fourier method gives an approximate solution in $N-1$ inner points with the maximum error $O\left(N^{-2}\right)$.

Proof. Let us express the exact solution $u(x)$ in the points $x_{\nu}=\nu / N, \nu=1$, $2, \ldots, N-1$, by its trigonometric interpolation polynomial,

$$
u(v / N)=\sum_{k=1}^{N-1} \widetilde{u}_{k} \sin (k \pi v / N)
$$

We intend to approximate $\widetilde{u}_{k}$ by $-\widetilde{F}_{k} / k^{2} \pi^{2}$. The difference between $F_{k}$ and the computed $\widetilde{F}_{k}$ is due to the so-called aliasing error (see [3]), which appears because the high frequencies cannot be distinguished when the data is discretized. Thus, for $\widetilde{F}_{k}$ we find the following relationship:

$$
\begin{aligned}
\widetilde{F}_{k} & =\frac{2}{N} \sum_{\nu=1}^{N-1} F\left(\frac{\nu}{N}\right) \sin (k \pi \nu / N) \\
& =\frac{2}{N} \sum_{\nu=1}^{N-1}\left(\sum_{p=1}^{\infty} F_{p} \sin (p \pi \nu / N)\right) \cdot \sin (k \pi \nu / N) .
\end{aligned}
$$

But $\sin (p \pi \nu / N)=\sin ((2 N r+p) \pi \nu / N)=-\sin ((2 N r-p) \pi \nu / N), r=1,2, \ldots$ We therefore obtain

$$
\sum_{p=1}^{\infty} F_{p} \sin (p \pi \nu / N)=\sum_{p=1}^{N-1}\left(F_{p}+\sum_{r=1}^{\infty}\left(F_{2 N r+p}-F_{2 N r-p}\right)\right) \cdot \sin (p \pi \nu / N),
$$

which by the orthogonality of the discrete sine series implies that

$$
\widetilde{F}_{k}=F_{k}+\sum_{r=1}^{\infty}\left(F_{2 N r+k}-F_{2 N r-k}\right)
$$


In the same way we obtain

$$
\tilde{u}_{k}=u_{k}+\sum_{r=1}^{\infty}\left(u_{2 N r+k}-u_{2 N r-k}\right) .
$$

Since $u_{k}=-F_{k} / k^{2} \pi^{2}$, we can then express $\tilde{u}_{k}$ in terms of $F_{k}$. Finally,

$$
\begin{aligned}
\delta_{k}=\tilde{u}_{k}-\hat{u}_{k}= & \sum_{r=1}^{\infty}-\left(\frac{F_{2 N r+k}}{\pi^{2}(2 N r+k)^{2}}-\frac{F_{2 N r-k}}{\pi^{2}(2 N r-k)^{2}}\right) \\
& +\frac{1}{\pi^{2} k^{2}} \sum_{r=1}^{\infty}\left(F_{2 N r+k}-F_{2 N r-k}\right) .
\end{aligned}
$$

The assumptions on the smoothness of $F(x)$ enable us to integrate by parts and derive an estimate for $F_{k}$, namely,

$$
F_{k}=-\frac{2}{\pi k}\left((-1)^{k} F(1)-F(0)\right)+o\left(\frac{1}{k^{2}}\right) .
$$

Using this expression for $F_{k}$, we find that $\delta_{k}=c(k) / k$, where $|c(k)| \leqslant M / N^{2}$ and $M$ is independent of $N$. The sum $\Sigma_{k=1}^{N-1} \sin (k \pi \nu / N) / k$ is uniformly bounded for $k, \nu$ and $N$. Using partial summation and the fact that $|c(k+1)-c(k)|=O\left(k / N^{4}\right)$, we can finally conclude that

$$
(\hat{u}(\nu / N)-u(\nu / N))=O\left(N^{-2}\right) \text { for } v=1,2, \ldots, N-1 . \quad \text { Q.E.D. }
$$

We will now show how this method can be modified to give better accuracy for more regular functions $F(x)$. The essential matter is to obtain a good estimate for the Fourier coefficients $F_{k}$. We introduce the following notation:

$$
f^{(p)}=\frac{\partial^{p} f(x)}{\partial x^{p}} \text { and } f^{(p, q)}=\frac{\partial^{p+q} f(x, y)}{\partial x^{p} \partial y^{q}} .
$$

Let us assume that $F \in C^{2 p-1}(0,1)$ and that $F^{(2 p)}$ is integrable. Repeated partial integration gives

$$
\begin{aligned}
F_{k}= & -\frac{2}{k \pi}\left(F(1)(-1)^{k}-F(0)\right)+\frac{2}{k^{3} \pi^{3}}\left(F^{(2)}(1)(-1)^{k}-F^{(2)}(0)\right) \\
& +\ldots+\frac{2(-1)^{p}}{(k \pi)^{2 p-1}}\left(F^{(2 p-2)}(1)(-1)^{k}-F^{(2 p-2)}(0)\right)+o\left(\frac{1}{k^{2 p}}\right)
\end{aligned}
$$

From this expression, we can derive an error estimate,

$$
(\hat{u}(\nu / N)-u(\nu / N))=O\left(N^{-2 p}\right),
$$

when $F^{(2 s)}(1)=F^{(2 s)}(0)=0, s=0,1, \ldots, p-2$.

As an example, let us consider a function $F(x)$ which is smooth enough to permit an error estimate $O\left(N^{-4}\right)$ had $F(0)$ and $F(1)$ been zero. If $F(x)$ differs from zero at $x=0$ and/or $x=1$, we proceed as follows. Let

$$
F(x)=x F(1)+(1-x) F(0)+F_{1}(x)
$$


where $F_{1}(0)=F_{1}(1)=0$. Consider the two boundary value problems

$$
\begin{aligned}
& u_{1}^{\prime \prime}(x)=F_{1}(x), \quad 0<x<1, \\
& u_{1}(0)=u_{1}(1)=0,
\end{aligned}
$$

and

$$
\begin{aligned}
& v_{1}^{\prime \prime}(x)=x, \quad 0<x<1, \\
& v_{1}(0)=v_{1}(1)=0 .
\end{aligned}
$$

The solution to the original problem (2.1) is then obtained as

$$
u(x)=u_{1}(x)+F(1) v_{1}(x)+F(0) v_{1}(1-x) .
$$

The Fourier method applied to (2.2) gives a solution with the accuracy $O\left(N^{-4}\right)$; and for (2.3), we have the explicit solution $v_{1}(x)=x\left(x^{2}-1\right) / 6$.

In a similar manner, we can obtain a sixth order method for any right-hand side $F(x)$ which is smooth enough. Starting with $F(x)$, we construct $F_{1}(x)$ as above. Then we write,

$$
F_{1}(x)=F_{2}(x)+F^{\prime \prime}(1) x\left(x^{2}-1\right) / 6+F^{\prime \prime}(0)(1-x)\left((1-x)^{2}-1\right) / 6 .
$$

Thus, $F_{2}(x)$ is sufficiently smooth and has the desired properties $F_{2}(0)=F_{2}(1)=$ $F_{2}^{\prime \prime}(0)=F_{2}^{\prime \prime}(1)=0$. This implies that the error estimate for the problem

$$
\begin{aligned}
& u_{2}^{\prime \prime}(x)=F_{2}(x), \quad 0<x<1, \\
& u_{2}(0)=u_{2}(1)=0,
\end{aligned}
$$

is $O\left(N^{-6}\right)$, when it is solved with our Fourier method. A fifth-degree polynomial $v_{2}$ can easily be found which satisfies

$$
\begin{aligned}
& v_{2}^{\prime \prime}(x)=x\left(x^{2}-1\right) / 6, \quad 0<x<1, \\
& v_{2}(0)=v_{2}(1)=0 .
\end{aligned}
$$

Thus, we obtain the solution as

$$
u(x)=F(1) v_{1}(x)+F(0) v_{1}(1-x)+F^{\prime \prime}(1) v_{2}(x)+F^{\prime \prime}(0) v_{2}(1-x)+u_{2}(x) .
$$

From this discussion, it should be evident how the procedure can be continued to give any accuracy provided $F(x)$ is sufficiently smooth.

3. The Two-Dimensional Case. We consider the problem

$$
\begin{aligned}
\Delta u=F & \text { in } \Omega, \\
u=0 & \text { on } \partial \Omega,
\end{aligned}
$$

where $\Omega$ is the unit square and $\partial \Omega$ is its boundary. We assume that $F^{(2,0)}, F^{(0,2)}$ and $F^{(1,1)}$ are of bounded total variation. The Fourier method can be described as follows. Let us extend $u(x, y)$ and $F(x, y)$ to be odd, 2-periodic functions in both $x$ and $y$. $F(x, y)$ can be represented as a Fourier series,

$$
F(x, y)=\sum_{k=1}^{\infty} \sum_{l=1}^{\infty} F_{k l} \sin (k \pi x) \sin (l \pi y),
$$


where $F_{k l}=4 \iint_{\Omega} F(x, y) \sin (k \pi x) \sin (l \pi y) d x d y$.

This formula also defines the periodic extension of $F$. Integrating by parts and using Eq. (3.1), we find

$$
-\frac{F_{k l}}{\pi^{2}\left(k^{2}+l^{2}\right)}=4 \iint_{\Omega} u(x, y) \sin (k \pi x) \sin (l \pi y) d x d y \equiv u_{k l} .
$$

We define the solution as

$$
u(x, y)=\sum_{k=1}^{\infty} \sum_{l=1}^{\infty} u_{k l} \sin (k \pi x) \sin (l \pi y),
$$

which is a uniformly convergent series if $F$ is smooth enough. Via the FFT, we compute approximations $\widetilde{F}_{k l}$ to $F_{k l}, k, l=1, \ldots, N-1$, sampling the values of $F(x, y)$ at $x=x_{\nu}=\nu / N, y=y_{\mu}=\mu / N, \nu, \mu=1, \ldots, N-1$. Thus, the right-hand side is replaced by its trigonometric interpolation polynomial,

$$
F(\nu / N, \mu / N)=\sum_{k=1}^{N-1} \sum_{l=1}^{N-1} \widetilde{F}_{k l} \sin (k \pi \nu / N) \sin (l \pi \mu / N) .
$$

The differential equation has the corresponding approximate solution

$$
u(\nu / N, \mu / N)=\sum_{k=1}^{N-1} \sum_{l=1}^{N-1}-\frac{\widetilde{F}_{k l}}{\pi^{2}\left(k^{2}+l^{2}\right)} \sin (k \pi \nu / N) \sin (l \pi \mu / N),
$$

which is evaluated at the meshpoints $(\nu / N, \mu / N)$ by an inverse FFT. We can now state a result which is analogous to Theorem 1 .

TheOREM 2. The Fourier method gives an approximate solution to Poisson's equation with homogeneous boundary data with an accuracy of $\left.\mathrm{O}_{(} \mathrm{N}^{-2}\right)$ in the meshpoints if

$$
F^{(2,0)}(x, 1), \quad F^{(2,0)}(x, 0), \quad F^{(0,2)}(1, y) \quad \text { and } \quad F^{(0,2)}(0, y)
$$

are of bounded total variation over $0 \leqslant x \leqslant 1$, respectively, $0 \leqslant y \leqslant 1$, and if $F^{(1,1)}$ is of bounded total variation over $0 \leqslant x \leqslant 1$ for every fixed $y, 0 \leqslant y \leqslant 1$, and if, finally, $\int_{0}^{1} F^{(1,1)}(x, y) \cos k \pi x d x$ is of bounded total variation, $0 \leqslant y \leqslant 1$.

Proof. The exact solution is given at the meshpoints by

$$
u(\nu / N, \mu / N)=\sum_{k=1}^{N-1} \sum_{l=1}^{N-1} \widetilde{u}_{k l} \sin (k \pi \nu / N) \sin (l \pi \mu / N),
$$

where $\tilde{u}_{k l}=u_{k l}+\mathrm{I}_{k l}(u)+\mathrm{I}_{l k}(u)+\mathrm{II}_{k l}(u)$ and $\mathrm{I}_{k l}$ and $\mathrm{II}_{k l}$ are defined by

$$
\mathrm{I}_{k l}(g)=\sum_{r=1}^{\infty}\left(g_{2 N r+k, l}-g_{2 N r-k, l}\right)
$$

and

$$
\mathrm{II}_{k l}(g)=\sum_{r=1}^{\infty} \sum_{s=1}^{\infty}\left(g_{2 N r+k, 2 N s+l}-g_{2 N r-k, 2 N s+l}-g_{2 N r+k, 2 N s-l}+g_{2 N r-k, 2 N s-l}\right) .
$$

This relationship is derived in the same way as the corresponding relation between $u_{k}$ and $\tilde{u}_{k}$ in the proof of Theorem 1. The Fourier method corresponds to approximating $\tilde{u}_{k l}$ by 


$$
\hat{u}_{k l}=-\widetilde{F}_{k l} / \pi^{2}\left(k^{2}+l^{2}\right),
$$

where $\widetilde{F}_{k l}=F_{k l}+\mathrm{I}_{k l}(F)+\mathrm{I}_{l k}(F)+\mathrm{II}_{k l}(F)$.

The exact relation between $u_{k l}$ and $F_{k l}$ is known and, therefore, $\tilde{u}_{k l}$ can be expressed in terms of $F_{k l}$. The difference, $\delta_{k l}=\tilde{u}_{k l}-\hat{u}_{k l}$, can then be estimated if the rate of decay of $F_{k l}$ is known. Integrating by parts, we find

$$
\begin{aligned}
F_{k l}= & \frac{4}{k l \pi^{2}}\left(F(0,0)-(-1)^{k} F(1,0)-(-1)^{l} F(0,1)+(-1)^{k+l} F(1,1)\right) \\
& -\frac{4}{k^{2} l \pi^{3}} \int_{0}^{1}\left(F^{(2,0)}(x, 0)-F^{(2,0)}(x, 1)(-1)^{l}\right) \cdot \sin k \pi x d x \\
& -\frac{1}{k l^{2} \pi^{3}} \int_{0}^{1}\left(F^{(0,2)}(0, y)-F^{(0,2)}(1, y)(-1)^{k}\right) \cdot \sin l \pi y d y \\
& +\frac{1}{k l \pi^{2}} \int_{0}^{1} \int_{0}^{1} F^{(1,1)}(x, y) \cos (l \pi y) \cos (k \pi x) d y d x .
\end{aligned}
$$

If a function $g(x)$ is of bounded total variation, one can show that

$$
\int_{0}^{1} g(x) \sin (k \pi x) d x=\frac{c}{k}+O\left(\frac{1}{k^{2}}\right) .
$$

Similarly, if $g(x, y)$ for every fixed $y$ is of bounded total variation, then $h_{k}(y) \equiv$ $\int_{0}^{1} g(x, y) \cos (k \pi x) d x=c(y) / k+O\left(1 / k^{2}\right)$ and if also $h_{k}(y)$ is of bounded total variation, then

$$
\int_{0}^{1} \int_{0}^{1} g(x, y) \cos (k \pi x) \cos (l \pi y) d x d y=O\left(\frac{1}{k l}\right)
$$

Therefore,

$$
\begin{aligned}
F_{k l}= & \frac{1}{k l}\left(A_{1}+A_{2}(-1)^{k}+A_{3}(-1)^{l}+A_{4}(-1)^{k+l}\right) \\
& +\frac{1}{k^{3} l}\left(B_{1}(k)+B_{2}(k)(-1)^{l}\right)+\frac{1}{k l^{3}}\left(C_{1}(l)+C_{2}(l)(-1)^{k}\right)+O\left(\frac{1}{k^{2} l^{2}}\right),
\end{aligned}
$$

where $A_{\nu}, \nu=1,2,3,4$, are bounded constants and $B_{1}, B_{2}$ and $C_{1}, C_{2}$ are uniformly bounded functions of $k$ and $l$, respectively. Let us insert $g_{k l}=1 / k l$ in $\mathrm{I}_{k l}(g)$. We obtain

$$
\mathrm{I}_{k l}(g)=\sum_{r=1}^{\infty} \frac{1}{l}\left(\frac{1}{2 N r+k}-\frac{1}{2 N r-k}\right)=\frac{1}{l} \sum_{r=1}^{\infty} \frac{-2 k}{4 N^{2} r^{2}-k^{2}}
$$

which gives $-\left(\pi^{2} / 9\right) k / l N^{2}<\mathrm{I}_{k l}(g)<-\left(\pi^{2} / 12\right) k / l N^{2}$. Using partial summation and the fact that $\Sigma_{k=1}^{N-1}(\sin k \pi \nu / N) / k$ is uniformly bounded, we can conclude that

$$
\sum_{k=1}^{N-1} \sum_{l=1}^{N-1} \frac{\mathrm{I}_{k l}(g)}{\left(k^{2}+l^{2}\right)} \cdot \sin k \pi \nu / N \sin l \pi \mu / N=O\left(\frac{1}{N^{2}}\right) .
$$

Analogous calculations for the other terms that contribute to $\delta_{k l}$ show that

$$
(\hat{u}(\nu / N, \mu / N)-u(\nu / N, \mu / N))=O\left(1 / N^{2}\right) \text {. Q.E.D. }
$$


This estimate can be improved if the coefficients $F_{k l}$ decay faster. Let us assume that $F^{(2,2)}$ exists and that $F$ is zero on the boundary. The three first terms in (3.2) would then be zero and the last term could be integrated once more to give

$$
F_{k l}=\frac{4}{k^{2} l^{2} \pi^{4}} \int_{0}^{1} \int_{0}^{1} F^{(2,2)}(x, y) \sin k \pi x \sin l \pi y d x d y .
$$

If $F^{(2,2)}$ satisfied the conditions imposed on $F$ in the statement of Theorem 2 we would be able to prove an error estimate $O\left(N^{-4}\right)$. Let us assume that $F$ is sufficiently smooth to allow an accuracy $O\left(N^{-4}\right)$ but that $F$ is not zero on the boundary. We then proceed as follows. Let us consider

$$
\begin{aligned}
F(x, y)= & F_{1}(x, y)+x y F(1,1)+x(1-y) F(1,0) \\
& +(1-x) y F(0,1)+(1-x)(1-y) F(0,0) .
\end{aligned}
$$

Then $F_{1}$ is zero at the corners. The solution $u(x, y)$ to Eq. (3.1) is obtained as

$$
\begin{aligned}
u(x, y)= & u_{1}(x, y)+v_{1}(x, y) F(1,1)+v_{1}(x, 1-y) F(1,0) \\
& +v_{1}(1-x, y) F(0,1)+v_{1}(1-x, 1-y) F(0,0),
\end{aligned}
$$

where $u_{1}(x, y)$ is the solution to the problem,

and $v_{1}(x, y)$ is the solution to

$$
\begin{array}{cc}
\Delta u_{1}=F_{1} & \text { in } \Omega, \\
u_{1}=0 & \text { on } \partial \Omega,
\end{array}
$$

$$
\begin{array}{cc}
\Delta v_{1}=x y & \text { in } \Omega, \\
v_{1}=0 & \text { on } \partial \Omega .
\end{array}
$$

The Fourier coefficients of $v_{1}$ are known explicitly. If $v_{1}$ is approximated by a partial sum of its Fourier series, the truncation error is $O\left(N^{-2}\right)$. Below we will propose a modified version of our method for the solution of the special corner problem (3.3). However, the main point is that $v_{1}$ is independent of $F$ and can therefore be computed once on a much finer mesh and then be stored on the coarser mesh.

The next step is to decompose $F_{1}$ as a sum of a function $F_{2}$, which is zero on the entire boundary, and a function $h(x, y)$ which depends in a simple way on the boundary values of $F_{1}$. Let

$$
h(x, y)=x F_{1}(1, y)+(1-x) F_{1}(0, y)+y F_{1}(x, 1)+(1-y) F_{1}(x, 0) .
$$

We can then write $F_{1}(x, y)=F_{2}(x, y)+h(x, y)$, where $F_{2}$ is zero on $\partial \Omega$. To find $u_{1}$, we must solve two new problems

$$
\begin{array}{cc}
\Delta u_{2}=F_{2} & \text { in } \Omega, \\
u_{2}=0 & \text { on } \partial \Omega,
\end{array}
$$

and

$$
\begin{aligned}
\Delta v_{2}=h & \text { in } \Omega, \\
v_{2}=0 & \text { on } \partial \Omega .
\end{aligned}
$$

The solution $u_{1}$ can be written as $u_{1}(x, y)=u_{2}(x, y)+v_{2}(x, y)$. The function $F_{2}$ is as smooth as the original function $F$. It is zero on $\partial \Omega$, and therefore the analysis above 
shows that the Fourier method applied to (3.4) gives an accuracy of $O\left(N^{-4}\right)$. The function $h(x, y)$ is a sum of functions $x h_{1}(y),(1-x) h_{2}(y), y h_{3}(x)$ and $(1-y) h_{4}(x)$, where $h_{s}(0)=h_{s}(1)=0, s=1,2,3$ and 4 . Let us consider the problem,

$$
\begin{aligned}
& \Delta w(x, y)=x h_{1}(y) \text { in } \Omega \text {, } \\
& w(x, y)=0 \quad \text { on } \partial \Omega \text {, }
\end{aligned}
$$

which we call a boundary problem. The function $h_{1}(y)$ can be extended to be an odd, 2-periodic function with a uniformly convergent Fourier series

where

$$
h_{1}(y)=\sum_{l=1}^{\infty} h_{l} \sin (l \pi y) \text {, }
$$

$$
h_{l}=2 \int_{0}^{1} h_{1}(y) \sin (l \pi y) d y .
$$

Make the Ansatz $w(x, y)=\Sigma_{l=1}^{\infty} s_{l}(x) \sin (l \pi y)$. We obtain a boundary value problem for each $s_{l}(x)$ which has the solution,

$$
s_{l}(x)=\frac{h_{l}}{l^{2} \pi^{2}}(\sinh (l \pi x) / \sinh (l \pi)-x) \equiv \frac{h_{l}}{l^{2} \pi^{2}}(T(l, x)-x) .
$$

The function $h_{1}(y)$ is as smooth as $F$ and furthermore $h_{1}(0)=h_{1}(1)=0$, which ensures that the coefficients $h_{l}$ decay like $1 / l^{3}$. The factor $(T(l, x)-x)$ is uniformly bounded. Therefore, the error estimate for the truncated series is $O\left(N^{-4}\right)$. When we use the approximate $\widetilde{h}_{l}$ as computed by FFT instead of $h_{l}$, the error in each coefficient is $O\left(l N^{-4}\right)$, and thus the total error is still $O\left(N^{-4}\right)$. The construction of $h(x, y)$ thus allows a decomposition into four simple problems, which depend only on boundary values of $F$, and for each of which the solution is obtained to an accuracy of $O\left(N^{-4}\right)$.

This procedure can be extended in the following way. Assume that $F^{(2 p, 2 p-1)}$ and $F^{(2 p-1,2 p)}$ are continuous, piecewise differentiable in the unit square and that $F^{(2 p, 2 p)}$ is integrable. Integrating by parts, we obtain an expansion for $F_{k l}$ in powers of $1 / k$ and $1 / l$,

$$
F_{k l}=\frac{A_{11}}{k l}+\frac{A_{21}}{k^{2} l}+\frac{A_{12}}{k l^{2}}+\frac{A_{33}}{k^{3} l^{3}}+\ldots+\frac{A_{2 p-1,2 p}}{k^{2 p-1} l^{2 p}}+\frac{A_{2 p, 2 p-1}}{k^{2 p} l^{2 p-1}}+\frac{c}{k^{2 p} l^{2 p}},
$$

where $A_{n n}$ depends on the corner values of $F^{(n-1, n-1)}, A_{n+1, n}$ depends on the boundary values of $F^{(n+1, n-1)}$ and $A_{n, n+1}$ depends on the boundary values of $F^{(n-1, n+1)}$. If $F^{(2 s, 2 s)}, s=0,1, \ldots, p-2$, all are zero on the boundary, the error estimate is $O\left(N^{-2 p}\right)$. Modifying the original procedure, we are able to construct a method with an accuracy of $O\left(N^{-2 p}\right)$ for any sufficiently smooth function $F$.

We have shown how to construct a function which is zero on the boundary for the case $p=2$. This construction led to one special corner problem and to four boundary problems. Assume that we know how to construct a function $F_{n}$, such that $F_{n}$ and its even derivatives up to the order $2(p-3)$ are zero on $\partial \Omega$. The next step is to decompose $F_{n}$ into a function $F_{n+1}$ such that $F_{n+1}^{(2 p-4,2 p-4)}$ is zero at the corners. This is achieved by the introduction of a function $q(x)$ such that $q^{(2 s)}(1)=q^{(2 s)}(0)=$ 0 for $s=0,1, \ldots, p-3, q^{(2 p-4)}(0)=0$ and $q^{(2 p-4)}(1)=1$. This function plays the role played by $x$ in the first step $(p=2)$. The function values at the corners used 
in the first step are now replaced by the values of $F_{n}^{(2 p-4,2 p-4)}$ at the corners. The new corner problem can be solved with our Fourier method with an error $O\left(N^{-2 p+2}\right)$ but is independent of the function $F$. From $F_{n+1}$, a function $F_{n+2}$ is constructed such that $F_{n+2}^{(2 p-4,2 p-4)}$ is zero at the boundary. Again $x$ from the first step is replaced by $q(x)$ and the boundary values of $F_{1}$ are replaced by the boundary values of $F_{n+1}^{(2 p-4,0)}$ etc. The boundary problem can be solved with an accuracy of $O\left(N^{-2 p}\right)$ if we apply the method proposed for Eq. (3.5). The remaining problem with $F_{n+2}$ as right-hand side can be solved with our Fourier method to an accuracy of $O\left(N^{-2 p}\right)$.

Let us now return to the first corner problem. We propose a special method similar to the method we have introduced for the boundary problems. The problem is defined by

$$
\begin{aligned}
\Delta v(x, y)=x y & \text { in } \Omega, \\
v(x, y)=0 & \text { on } \partial \Omega .
\end{aligned}
$$

The solution can be written as

$$
v(x, y)=\sum_{k=1}^{\infty} \sum_{l=1}^{\infty} \frac{-4(-1)^{k+l}}{k l\left(k^{2}+l^{2}\right) \pi^{4}} \sin (k \pi x) \sin (l \pi y) .
$$

The truncation error is $O\left(N^{-2}\right)$ if we take a partial sum with $k, l=1,2, \ldots, N-1$. We notice that this problem is of the same kind as (3.5) only that we have replaced $h_{1}(y)$ by $y$. Therefore, we may also write

$$
v(x, y)=\sum_{l=1}^{\infty} \frac{-2(-1)^{l}}{l^{3} \pi^{3}} \cdot(T(l, x)-x) \sin (l \pi y) .
$$

Recognizing $2(-1)^{l} / l^{3} \pi^{3}$ as the Fourier coefficient for $\left(y^{3}-y\right) / 6$, we put

$$
v(x, y)=x\left(y^{3}-y\right) / 6+\sum_{l=1}^{\infty} \frac{-2(-1)^{l}}{l^{3} \pi^{3}} T(l, x) \sin (l \pi y) .
$$

Furthermore, $x$ and $y$ may be interchanged everywhere. We can thus write $v(x, y)$ in a symmetric form. The truncation error of this series is still $O\left(N^{-2}\right)$. However, we are able to improve this considerably at least for some values of $x$ and $y$ if we estimate $T(l, x)$ more carefully. The function $T(l, x)=\sinh (l \pi x) / \sinh (l \pi)$ decays like $e^{(x-1) l \pi}$, $0<x<1$. Thus, we can save computing time by using different values of $N$ for different values of $x$. As an example, let us assume that we want the solution with an error of less than $10^{-6}$ for $x=\nu / 32, \nu=1,2, \ldots, 31$. For $\nu \leqslant 28$, we can estimate the truncation error by $N^{-2} e^{-N \pi / 8} / \pi^{3}$, which for $N=32$ is of the order $10^{-9}$. For $\nu=29,30,31$, we should use $N=64$.

We conclude the discussion of the Dirichlet problem with the construction of a function $w$, which satisfies the boundary condition $w=g$ on $\partial \Omega$. If $g$ is known analytically, we can simply take

$$
w(x, y)=(1-x) g(0, y)+x g(1, y)+(1-y) g(x, 0)+y g(x, 1)
$$

and

$$
\Delta w(x, y)=(1-x) g^{(0,2)}(0, y)+x g^{(0,2)}(1, y)+(1-y) g^{(2,0)}(x, 0)+y g^{(2,0)}(x, 1) .
$$


When $g$ is known only pointwise, we use trigonometric interpolation. Let us consider $g(0, y)$. We write

$$
g_{1}(0, y)=g(0, y)-(1-y) g(0,0)-y g(0,1)
$$

and we then compute the coefficients $\left(\widetilde{g}_{1}\right)_{l}$ in the trigonometric interpolation polynomial for $g_{1}(0, y)$,

$$
g_{1}(0, \mu / N)=\sum_{l=1}^{N-1}\left(\widetilde{g}_{1}\right)_{l} \sin (l \pi \mu / N)
$$

Trigonom iric interpolation polynomials are constructed in a similar way for the other boundaries. The function $w(x, y)$ can now be written as

$$
\begin{aligned}
w(x, y)= & (1-x)(1-y) g(0,0)+(1-x) y g(0,1)+x(1-y) g(1,0)+x y g(1,1) \\
& +(1-x) g_{1}(0, y)+x g_{1}(1, y)+(1-y) g_{1}(x, 0)+y g_{1}(x, 1) .
\end{aligned}
$$

We also need $\Delta w$ so we must compute, for example, $g_{1}^{(0,2)}(0, y)$. Term-by-term differentiation of the trigonometric interpolation polynomial for $g_{1}(0, y)$ gives

$$
\hat{g}_{1}^{(0,2)}(0, \mu / N)=\sum_{l=1}^{N-1}-l^{2} \pi^{2}\left(\widetilde{g}_{1}\right)_{l} \sin (l \pi \mu / N) .
$$

By construction, $g_{1}(0,0)=g_{1}(0,1)=0$ and the Fourier coefficients for $g_{1}(0, y)$, therefore, decay at least like $1 / l^{3}$ and those for $g_{1}^{(0,2)}(0, y)$ decay like $1 / l$. The difference between $-l^{2} \pi^{2}\left(\sigma_{1}\right)$ and the exact coefficient in the interpolation polynomial for $g_{1}^{(0,2)}(0, y)$ is $O\left(l / N^{2}\right)$. The influence on the solution can be shown to be $O\left(1 / N^{2}\right)$, i.e., this construction maintains the second order accuracy. To obtain fourth order accuracy we need a better approximation of the boundary values $g$. This requires information about the second derivatives of $g$ in the corners.

4. Neumann Boundary Condition. We will briefly discuss the application of the Fourier technique to the Neumann problem

$$
\begin{aligned}
\Delta u=F & \text { in } \Omega, \\
\partial u / \partial n=0 & \text { on } \partial \Omega .
\end{aligned}
$$

The arguments in this case are similar to those for the Dirichlet problem if we replace the sine-expressions with cosines. Let us extend $u$ and $F$ to be even 2-periodic functions. Then we may write

$$
u(x, y)=\sum_{k=0}^{\infty} \sum_{l=0}^{\infty} u_{k l} \cos (k \pi x) \cos (l \pi y),
$$

where

$$
u_{k l}=4 \int_{0}^{1} \int_{0}^{1} u(x, y) \cos (k \pi x) \cos (l \pi y) d x d y .
$$

Integrating by parts and using Eq. (4.1), we find

$$
-\left(k^{2}+l^{2}\right) \pi^{2} u_{k l}=4 \int_{0}^{1} \int_{0}^{1} F(x, y) \cos (k \pi x) \cos (l \pi y) d x d y \equiv F_{k l} .
$$

Green's identity implies that $F_{00}=4 \int_{0}^{1} \int_{0}^{1} F(x, y) d x d y=0$. As (4.1) only determines $u$ modulo a constant, we can in addition prescribe $u$ at one point and use $u_{00}$ to obtain that particular solution. Let us represent $u(\nu / N, \mu / N)$ and $F(\nu / N, \mu / N), \nu, \mu=0$, $1, \ldots, N-1$, by their trigonometric interpolation polynomials 


$$
u(\nu / N, \mu / N)=\sum_{k=0}^{N-1} \sum_{l=0}^{N-1} \tilde{u}_{k l} \cos (k \pi \nu / N) \cos (l \pi \mu / N)
$$

and

$$
F(\nu / N, \mu / N)=\sum_{k=0}^{N-1} \sum_{l=0}^{N-1} \widetilde{F}_{k l} \cos (k \pi \nu / N) \cos (l \pi \mu / N) .
$$

Generally, the coefficients $\widetilde{g}_{k l}$ in the trigonometric interpolation polynomial for $g(x, y)$ using only cosines are related to the Fourier coefficients $g_{k l}$ as

$$
\widetilde{g}_{k l}=g_{k l}+\mathrm{I}_{k l}(g)+\mathrm{I}_{l k}(g)+\mathrm{II}_{k l}(g),
$$

where

$$
\mathrm{I}_{k l}(g)=\sum_{r=1}^{\infty}\left(g_{2 N r+k, l}+g_{2 N r-k, l}\right)
$$

and

$$
\begin{aligned}
\mathrm{II}_{k l}(g)=\sum_{r=1}^{\infty} \sum_{s=1}^{\infty}\left(g_{2 N r+k, 2 N s+l}+g_{2 N r+k, 2 N, s-l}\right. \\
\left.+g_{2 N r-k, 2 N s+l}+g_{2 N r-k, 2 N s-l}\right) .
\end{aligned}
$$

We intend to approximate $\tilde{u}_{k l}$ by $-\widetilde{F}_{k l} /\left(k^{2}+l^{2}\right) \pi^{2}$. The error we make can be expressed in terms of $F_{k l}$. Assuming that $F$ is smooth enough, we integrate by parts and obtain for $k \neq 0, l \neq 0$

$$
\begin{aligned}
F_{k l} & =4 \int_{0}^{1} \int_{0}^{1} F(x, y) \cos (k \pi x) \cos (l \pi y) d x d y \\
& =\frac{4}{k l \pi^{2}} \int_{0}^{1} \int_{0}^{1} F^{(1,1)}(x, y) \sin (k \pi x) \sin (l \pi y) d x d y,
\end{aligned}
$$

from where we can continue as in (3.2). For $k \neq 0, l=0$ we obtain

$$
\begin{aligned}
F_{k 0}= & 4 \int_{0}^{1} \int_{0}^{1} F(x, y) \cos (k \pi x) d x d y \\
= & \frac{4}{k^{2} \pi^{2}} \int_{0}^{1}\left((-1)^{k} F^{(1,0)}(1, y)-F^{(1,0)}(0, y)\right) d y \\
& \quad-\frac{4}{k^{2} \pi^{2}} \int_{0}^{1} \int_{0}^{1} F^{(2,0)}(x, y) \cos k \pi x d x d y .
\end{aligned}
$$

The estimate $F_{k l}=O\left(1 / k^{2} l^{2}\right) \cdot\left(O\left(1 / k^{2}\right)\right.$ or $O\left(1 / l^{2}\right)$ for $l$ or $k$ zero) gives an error estimate $O\left(1 / N^{2} k^{2} l^{2}\right)$ in each coefficient $\left(O\left(1 / N^{2} k^{2}\right)\right.$ respectively $\left.O\left(1 / N^{2} l^{2}\right)\right)$. Since $\Sigma_{k=1}^{N} \cos k \pi x / k^{2}$ is uniformly bounded, we can conclude that the total error is $O\left(N^{-2}\right)$. If $F$ is regular enough, more accurate solutions can be achieved by procedures, similar to those described in Section 3, involving special solutions to corner and boundary problems.

5. Results. Numerical experiments were carried out to demonstrate the order of accuracy of our Fourier method. Comparisons were made with the Buneman algorithm for the standard five-point formula and a fourth order accurate ninepoint formula to obtain some information on the efficiency of the Fourier method. As 
a test example, we chose the equation

$$
\Delta u(x, y)=-2 \alpha^{2} \pi^{2} \sin (\alpha \pi(x+y))
$$

with Dirichlet boundary data and the solution $u(x, y)=\sin (\alpha \pi(x+y))$. The solution has rapidly growing derivatives for large values of $\alpha$, which is directly reflected in the maximum error when finite differences are used. The solution with the Fourier method also show : an increasing error with increasing $\alpha$. However, the rate of increase seems to be son, :what less than for the finite difference solution. In the tables below, $N$ denotes the number of meshpoints (including one boundary) in each direction.

\begin{tabular}{cccccc}
\hline & $\alpha$ & $\begin{array}{c}\text { Second order } \\
\text { Fourier method }\end{array}$ & $\begin{array}{c}\text { Five-point } \\
\text { formula }\end{array}$ & $\begin{array}{c}\text { Fourth order } \\
\text { Fourier method }\end{array}$ & $\begin{array}{c}\text { Nine-point } \\
\text { formula }\end{array}$ \\
\hline & & $7.8 \cdot 10^{-4}$ & $4.0 \cdot 10^{-4}$ & $8.5 \cdot 10^{-7}$ & $1.2 \cdot 10^{-7}$ \\
32 & 5 & $1.6 \cdot 10^{-2}$ & $2.3 \cdot 10^{-2}$ & $4.5 \cdot 10^{-4}$ & $1.8 \cdot 10^{-4}$ \\
& 9 & $5.2 \cdot 10^{-2}$ & $7.6 \cdot 10^{-2}$ & $4.7 \cdot 10^{-3}$ & $1.8 \cdot 10^{-3}$ \\
\hline & 1 & $2.0 \cdot 10^{-4}$ & $1.0 \cdot 10^{-4}$ & $5.6 \cdot 10^{-8}$ & $7.8 \cdot 10^{-9}$ \\
64 & 5 & $4.6 \cdot 10^{-3}$ & $5.7 \cdot 10^{-3}$ & $3.1 \cdot 10^{-5}$ & $1.1 \cdot 10^{-5}$ \\
9 & $1.3 \cdot 10^{-2}$ & $1.9 \cdot 10^{-2}$ & $3.0 \cdot 10^{-4}$ & $1.2 \cdot 10^{-4}$
\end{tabular}

TABLE 1. The maximum error for the solution to the equation, $\Delta u=-2 \alpha^{2} \pi^{2} \sin (\alpha \pi(x+y))$. Two second order methods and two fourth order methods were used.

In the example above, the solution is very well behaved, and we need not worry about any corner problems because the function values are zero at the corners. Let us also consider the first corner problem,

$$
\begin{aligned}
\Delta u=x y & \text { in } \Omega, \\
u=0 & \text { on } \partial \Omega .
\end{aligned}
$$

The solution was computed to an accuracy of $10^{-12}$ in $32 \times 32$ meshpoints. We compare the results obtained with different methods using $N$ meshpoints.

\begin{tabular}{ccccc}
\hline$N$ & $\begin{array}{c}\text { Second order } \\
\text { Fourier method }\end{array}$ & $\begin{array}{c}\text { Five-point } \\
\text { formula }\end{array}$ & $\begin{array}{c}\text { Special } \\
\text { method }\end{array}$ & $\begin{array}{c}\text { Nine-point } \\
\text { formula }\end{array}$ \\
\hline 32 & $8.4 \cdot 10^{-5}$ & $2.5 \cdot 10^{-5}$ & $1.1 \cdot 10^{-7}$ & $1.4 \cdot 10^{-6}$ \\
64 & $1.8 \cdot 10^{-5}$ & $6.4 \cdot 10^{-6}$ & $7.6 \cdot 10^{-10}$ & $8.5 \cdot 10^{-8}$ \\
\hline
\end{tabular}

TABLE 2. The maximum error for the solution to the equation, $\Delta u=x y$ in $\Omega, u=0$ on $\partial \Omega$. The error is measured in $32 \times 32$ points (also for $N=64$ ).

The special method refers to the method proposed at the end of Section 3 for the first corner problem. The error estimates given there are seen to be too pessimistic. The use of the fourth order Fourier method would mean the use of the tabulated solution, and thus, give an error of less than $10^{-12}$ in this case. 
With homogeneous boundary data, the solution of the finite difference equations is about $20 \%$ faster than the solution with our Fourier method. For inhomogeneous data, the Fourier method requires additional work. The nine-point program which was used also requires a considerable amount of overhead to handle the data. We have therefore chosen to give the time estimates both for the "equation solver" without overhead and the total time with everything included. We give the average time in seconds. We believe that these numbers are correct to within $5 \%$.

\begin{tabular}{ccccc}
\hline$N$ & \multicolumn{2}{c}{$\begin{array}{c}\text { Second order } \\
\text { Fourier method }\end{array}$} & \multicolumn{2}{c}{ Five-point formula } \\
\hline & Eq. solver & Total & Eq. solver & Total \\
\cline { 2 - 5 } 32 & 0.72 & 1.10 & 0.60 & 0.74 \\
\hline 64 & 3.50 & 4.75 & 2.90 & 3.24 \\
\hline
\end{tabular}

TABLE 3. Time in seconds for the second order methods.

\begin{tabular}{ccccc}
\hline$N$ & \multicolumn{2}{c}{$\begin{array}{c}\text { Fourth order } \\
\text { Fourier method }\end{array}$} & Nine-point formula \\
\hline & Eq. solver & Total & Eq. solver & Total \\
\cline { 2 - 5 } 32 & 1.10 & 2.25 & 0.96 & 2.60 \\
\hline 64 & 5.45 & 9.70 & 4.50 & 12.00 \\
\hline
\end{tabular}

TABLE 4. Time in seconds for the fourth order methods.

One should note that the most expensive part of the fourth order Fourier method is the final FFT, which is used to compute the solution of the boundary problem in every point given the series expansions,

$$
\sum_{l=1}^{N-1} s_{l}(\nu / N) \sin (l \pi \mu / N) \text { and } \sum_{k=1}^{N-1} s_{k}(\mu / N) \sin (k \pi \nu / N)
$$

One of these must be performed by itself while the other one can be incorporated with the straightforward Fourier solution for the smooth problem. This additional FFT accounts for the $50 \%$ increase in time between the second and fourth order "equation solvers". For higher order methods there are several contributions from boundary problems, but they may all be computed with the same FFT and the additional work increase is comparatively small. All test runs have been made on the IBM 370/155 computer at Uppsala University Data Center, using the FORTRAN II G1 compiler.

The main advantage of our Fourier method is perhaps the simple algorithm for the straightforward second order solution. The method is efficient if an efficient FFT subroutine is available. Higher order methods require much knowledge of the bound- 
ary data. The fourth order methods only involve the function values of the right-hand side and, therefore, seem to be realistic to use.

Department of Computer Sciences

Uppsala University

Sturegatan 4B 2tr

S-752-23 Uppsala, Sweden

1. O. BUNEMAN, A Compact Non-Iterative Poisson Solver, Rep. 294, Stanford University, Institute for Plasma Research, Stanford, Calif., 1969.

2. B. L. BUZBEE, G. H. GOLUB \& C. W. NIELSON, "On direct methods for solving Poisson's equations," SIAM J. Numer. Anal., v. 7, 1970, pp. 627-656. MR 44 \#4920.

3. J. W. COOLEY, P. A. W. LEWIS \& P. D. WELCH, "The fast Fourier transform and its applications," IEEE Trans. Education, v. E-12, 1969, pp. 27-34.

4. R. W. HOCKNEY, "The potential calculation and some applications," Methods in Computational Physics, v. 9, 1970, pp. 135-211.

5. J. B. ROSSER, Fourier Series in The Computer Age, Mathematics Research Center, Technical Summary Report \#1401, University of Wisconsin, Madison, Wis., Feb. 1974. 